East African Medical Journal Vol. 80. No. 8 August 2003

TRADITIONAL REMEDIES IN CHILDREN AROUND EASTERN CAPE, SOUTH AFRICA

Y.M. Dambisya, MBChB, PhD, Professor, School of Health Sciences, University of the North, Sovenga 0727 and G. Tindimwebwa, MBChB, MMed, Professor, Department of Paediatrics, University of Transkei Medical School, UNITRA 5117, Republic of South Africa

Request for reprints to: Prof. Y. M. Dambisya, Pharmacy Programme, School of Health Sciences, University of the North, Private Bag X1106, Sovenga, 0727, Republic of South Africa

\title{
TRADITIONAL REMEDIES IN CHILDREN AROUND EASTERN CAPE, SOUTH AFRICA
}

\author{
Y.M. DAMBISYA and G. TINDIMWEBWA
}

\begin{abstract}
Objective: To gauge the views of mothers and other caregivers on the use of traditional remedies in the community.

Design: Descriptive study.

Setting: Five health centres affliated to the UNITRA, Faculty of Health Sciences.

Methods: Medical students administered a questionnaire to mothers and other caregivers. Areas covered included how commonly traditional remedies are used, experience with traditional remedies in children, reasons for use, how administered, and problems encountered. Results: There were 103 respondents, $7.8 \%$ rated use of traditional remedies non-existent, $20.4 \%$ rated it as rare and the rest said it was common. $57.3 \%$ of the respondents had used these remedies in their children, and $83.1 \%$ of them said they would use them again. Only $20.7 \%$ of respondents reported problems with these remedies, including diarrhoea, vomiting, fever and death. The preparations used were known by only $40.7 \%$ of respondents. The remedies were administered by the mother and grandmother in $82.8 \%$ of cases; and were mainly given orally, by enema or by scarification. The amount administered varied from spoonful(s) up to $2.5 \mathrm{l}$. Children of all ages were given traditional remedies, usually for diarrhoea, fevers and constipation, or as prophylactics. These remedies were used because they are effective, available and safe, or due to customary beliefs and pressure from others. Conclusion: The use of traditional remedies in children is common in communities around Umtata. There is need to study the composition and activity profiles of commonly used preparations so as to develop appropriate management protocols in case of toxicity.
\end{abstract}

\section{INTRODUCTION}

It is widely held that the use of traditional remedies is common in Africa, and that many patients who visit traditional healers do not need to resort to Western Medicine, and that many patients presenting to the health units will have tried traditional remedies first $(1,2)$. Traditional remedies are a popular choice of health care partly because they are culturally acceptable and readily available in the rural communities $(1,3)$. These preparations (especially herbal remedies) have become increasingly popular as alternative medicines in the developed world too.

The fact that the benefits of traditional remedies remain largely unproven by the scientific method and the concern about adverse effects have led to closer scrutiny of these products (4). Whereas most traditional remedies are safe, the potential for adverse effects or intoxication exists, as does the possibility of interaction with conventional drugs $(2,5-8)$. All these unwanted effects are even more likely to occur in children, given their special pharmacokinetics (9).

The Umtata General Hospital Paediatric Out-patient' Department attends to quite a number of children in whom a recent history of traditional medication can be elicited.
Many of them are admitted as "herbal intoxication", and it has been shown that about $40 \%$ of patients admitted with a herbal intoxication label die (10). As part of an ongoing study into the use and toxicity of traditional remedies in children we did a survey of mothers and other caregivers of children brought to five health centres that are part of the University of Transkei (UNITRA), Faculty of Health Sciences Teaching complex. This was done in the hope that the views of these caregivers would help us understand the profile of traditional remedy use in the community. A preliminary account of this work was presented at the 4th Eastern Cape Health Research Conference (11).

\section{MATERIALS AND METHODS}

The study was conducted at the five health centres of Baziya, Mbekweni, Stanford Terrace, Muhlakulo, and Ngangelizwe, which are Community-Higher Education Service Partnership (CHESP) units that our faculty uses for communitybased education and service (COBES) activities (12). In addition, these centres were chosen because they refer many of the children admitted to our wards as herbal intoxication .

Third year medical students administered a questionnaire to mothers and other caregivers with children at the health centres. Participation in the survey (by both students and 
respondents) was voluntary, and verbal consent was obtained from all respondents. The questionnaire included questions on the respondents' perceptions on how commonly traditional remedies are used in the community and what problems are associated with that practice; and questions on personal experience with use of these preparations in children. Those who admitted using traditional remedies in their children were asked whether or not they would use them again and whether or not they would recommend them to others. In addition, the respondents were asked about the source of the preparations, who administers them to the children, the name(s) of the preparations and how much is given.

The results were compiled and summary statistics generated using MS Excel. The data are presented as frequencies or percentages of the responses obtained.

\section{RESULTS}

There were 103 completed questionnaires. Some mothers and caregivers declined to participate in the study on the grounds that use of traditional remedies was against their religious beliefs.

How common is the use of traditional remedies?: Of the 103 respondents, eight $(7.8 \%)$ said there is no use of traditional remedies in their community. As shown in Table Ia most respondents stated that use of these remedies is common in the community, with $43.7 \%$ saying the practice is common or very common. In addition, $57.2 \%$ of respondents admitted using traditional remedies in their own children within the last one year or so (Table Ib).

Table 1

How commonly used are traditional remedies?

(a) Respondents perceptions on how commonly used traditional remedies are in the community

\begin{tabular}{lll} 
& No. of responses & $\%$ \\
\cline { 2 - 3 } No answer & 3 & 2.9 \\
Not used at all & 8 & 7.8 \\
Rarely & 21 & 20.4 \\
Happens & 26 & 25.2 \\
Common & 28 & 27.2 \\
Very common & 17 & 16.5
\end{tabular}

(b) Respondents experiences with the use traditional remedies in children

\section{No answer}

Have never used

Used in the last 1 year or earlier

Used in the last six months

Used in the last 1 month

Used in the last 1 week
No. of responses

$\%$

$\begin{array}{ll}2 & 1.9 \\ 42 & 40.8 \\ 30 & 29.1 \\ 10 & 9.7 \\ 9 & 8.7 \\ 10 & 9.7\end{array}$

Among those who have used traditional remedies in children, 49 out of $59(83.1 \%)$ said they would use them again, eight $(13.6 \%)$ said they would not, while two respondents $(3.3 \%)$ gave no answer. Of those who stated that they had never used traditional remedies in their children $(n=42)$, eight $(19.0 \%)$ said they would use them, as did the two respondents who did not indicate whether or not they had used traditional remedies in their children. Overall then, 59 out of $103(57.3 \%)$ said they would use them, compared to $38(36.9 \%)$ who said they would not (including 10 who have used before), and six (5.8\%) did not answer.

When asked whether or not they would encourage others to use traditional remedies in children, 54 answered in the affirmative: 10 out of $42(23.8 \%)$ who have never used, 42 out of $59(71.2 \%)$ who have used in their own children, and two who gave no answer on previous use. Of the rest, 42 said they would not encourage others, and seven did not answer the question. 
Table 2

Indications and routes of administration of traditional remedies in children

(a) Indication

\begin{tabular}{lll} 
& No. of responses & $\%$ \\
\cline { 2 - 3 } Diarrhoea & 47 & 29.4 \\
Fevers & 39 & 24.4 \\
As Prophylactics & 22 & 13.8 \\
Constipation & 8 & 5.0 \\
Stomachache & 7 & 4.4 \\
Cough and flu & 6 & 3.8 \\
Plate & 5 & 3.1 \\
Evil spirits/witchcraft & 4 & 2.5 \\
Cancer & 3 & 1.9 \\
To induce vomiting & 2 & 1.3 \\
Seizures & 2 & 1.3 \\
Thrush & 2 & 1.3 \\
Others* & 13 & 8.2
\end{tabular}

(b) Why traditional remedies?

\begin{tabular}{lll} 
& No. of responses & $\%$ \\
\cline { 2 - 3 } Effective & 63 & 32.6 \\
Available & 34 & 17.6 \\
Customary requirements/beliefs & 32 & 16.6 \\
Safe & 31 & 16.1 \\
Lack of alternative & 19 & 9.8 \\
Pressure/advice from others & 9 & 4.7 \\
Others** & 5 & 2.6 \\
\hline
\end{tabular}

*Other indications-One each of dysmennorrhoea, TB, worms, pain, headache, pubic lice, arthritis, backache, measles, teething, nyongo, infertility and food poisoning.

**Other reasons (number of responses): Witchcraft (2), against evil spirits (2), failed western medicine (1).

Why are these remedies used?: Table 2a shows the most commonly cited indications for the use of traditional remedies in children. Most often they are used to treat preexisting common childhood illnesses (diarrhoea, fevers). As shown in Table $2 \mathrm{~b}$, these remedies are used because they are thought to be safe $(16.1 \%)$, effective $(32.6 \%)$, readily available $(17.6 \%)$; for lack of alternatives $(9.8 \%)$, due to pressure from or on the advice of others $(4.7 \%)$. Customary practices/beliefs also account significantly to the use of these remedies

Administration: The age group(s) of children given traditional remedies were: all ages $11.6 \%, 6$ months or younger $26.7 \%, 6$ months to 1 year $25.6 \%, 1$ to 5 years $12.8 \%, 5$ to 12 years $12.8 \%$ and 12 years or older $10.5 \%$. The remedies are administered by the mother $(71.7 \%)$, grandmother (11.1\%), herbalist/traditional healer (15.2\%), or father $(2.0 \%)$. In the majority of cases, they are given orally $(50 \%)$, by enema $(27.6 \%)$, or by scarification $(17.1 \%)$. Less frequently cited routes of administration were sniffing, wearing around the neck, body wash, smearing, and throat/mouth wash. The measure of the amount(s) given to the children was estimated in terms of spoonful $(46.7 \%)$, cupful $(41.1 \%)$, bottle $(8.9 \%)$, and others, up to $2.5 \mathrm{~L}(3.3 \%)$.

What is given and where is it from?: About half the respondents (48\%) did not know what the remedies were, and many of the others only knew the preparation(s) in a generic sense, e.g.iyeza le plate which is literary "medicine for plate", ilitye lamgodi (medicine from the soil). The commonest preparations were: Iyeza le plafe $(21.4 \%)$, Umhlonyane (28.6\%), Qwili (7.1\%), andImpepho (7.1\%). The source of the traditional remedies was herbalists or traditional doctor (Inyanga) $(55.7 \%)$, the shop or vendor or chemist $(23.9 \%)$, other members of the community (3.4\%), or the mothers/caregivers obtain it for themselves from the bush/garden/veld (17.0\%).

Problems with traditional remedies?: Among those who have used these remedies in their children $12(20.3 \%)$ said they had experienced some problems, while $47(79.7 \%)$ reportedly had no problems. Overall, $24.1 \%$ of respondents said they were aware of problems associated with traditional remedies, while $75.9 \%$ were not aware of any problems. The problems cited included death (eight responses), dehydration (three responses), diarrhoea (three responses), fever (three responses), poisoning/overdose (two responses), epilepsy (two responses), and skin rash, oedema, weakness, and vomiting (each cited once).

\section{DISCUSSION}

The present study shows that there is widespread acceptance and use of traditional remedies in children in communities around Umtata. One of the limitations of the study is the fact that it was based at health centres and targeted those who sought the services at those centres. We may therefore have missed the views of those who use traditional remedies and do not come to the health centres. Secondly, the veracity of the responses given in that setting may be suspect since some may not have wanted to say anything offensive to the "medical" establishment. The real figures, for instance on personal experience with use of the remedies in children, may be much higher than obtained in the survey. However, the medical students identified themselves as such, and made it clear that the respondents were free to answer the questions or to decline, and even more so that the care given to their children would not be influenced by their decision in this regard.

That most respondents were not aware of any problems associated with traditional remedies could be explained in a number of ways. In many instances, these remedies are given for a pre-existing medical problem, so when the child gets worse it would be difficult to know whether the deterioration was due to the primary condition or due to the medication. This is a problem we face when dealing with children admitted for "herbal intoxication" (10). The other possibility may be that the preparations given are safe, for a practice that widespread is unlikely to survive in the face of many common problems. Those that get to the hospital may be the extreme cases, hence the high case 
fatality rate observed in the Paediatric Department of Umtata General Hospital (10). Nevertheless, the fact that some of the respondents attributed adverse effects, including death, to the use of traditional remedies shows that even the community is aware of the harmful effects of some of these remedies.

The age of children given the remedies, and the amount(s) thereof administered raise a lot of concern, given the relative intolerance of the neonates and young infants to most preparations (9). This is borne out by our experience where most admissions and deaths due to "herbal intoxication" occur among neonates and infants below six months (10). The problem is compounded by the fact that the identity or contents of the preparations are unknown to the mothers in the majority of cases, and even when known they are only known in a descriptive sense. Since many of the mothers and caregivers obtain the traditional remedies from the shop or traditional healers (Inyangas), that offers a starting point for the elucidation of these preparations. In furtherance of the present study, plans are underway to enlist the cooperation of some of the traditional healers to obtain common remedies for scientific analysis.

The reasons cited for the use of the remedies, for instance safety, availability and accessibility, are similar to those cited elsewhere $(1,3)$. Even if it were found that all traditional remedies are harmful, their use would only stop with the ready availability of alternatives. In the absence of any alternatives, the mothers are compelled to use whatever is available (1).

That traditional remedies have beneficial effects seems well-established, but so are their deleterious effects $(13,14)$. The problem is to know which ones are beneficial, which ones are harmless, and which ones are harmful. The practitioners and consumers of these preparations need to exercise more caution when it comes to the administration of such preparations to children, given the special pharmacokinetics of most xenobiotics in children (9). Even without knowing the exact contents, we are in the process of working out the clinical profile(s) of the commonest modes of intoxication seen in children in our wards, with a view to establishing a rapid-response protocol. The need to study the composition and activity profiles of the most commonly used preparations so that appropriate management protocols can be put in place in case of toxicity/overdosing is self-evident.

\section{ACKNOWLEDGEMENTS}

This work was done while Dr. Y.M. Dambisya was at the Department of Pharmacology, Faculty of Health Sciences, UNITRA. We wish to thank the mothers and other caregivers who agreed to complete the questionnaire, the third year medical students (2002) who administered the questionnaire, and S. Mdludlu, and T. Boti, our student research assistants for their help in data handling. This study was funded by a Research Grant from UNITRA.

\section{REFERENCES}

1. Campbell, S. Traditional medicine in The Gambia. Complement. Ther. Nurs. Midwifery. 1997; 3:103-105.

2. Luyckx, V.A., Ballantine, R., Claeys, M., et al. Herbal remedyassociated acute renal failure secondary to Cape aloes. Am. J. Kidney. Dis. 2002; 39:E13.

3. Hibler, M. From hospitals to herbalists: Rx herbal medicines. Science in Africa Online.http:/www.scienceinafrica.co.za/2001/ november/herbs.htm.

4. Chitturi, S. and Farrell, G.C. Herbal hepatotoxicity: an expanding but poorly defined problem. J. Gastroenterol. Hepatol. 2000; 15:1093-1099.

5. Escher, M., Desmeules, J., Giostra E. and Mentha, G. Hepatitis associated with Kava, a herbal remedy for anxiety. Brit. Med. J. 2001; 322:139.

6. Anderson, N.R., Gama, R. and Kapadia, S. Herbal remedy poisoning presenting with acute abdomen and raised urine porphyrins. Ann. Clin. Biochem. 2001; 38:408-410.

7. Pinn, G. Adverse effects associated with herbal medicine. Aust. Fam. Physician. 2001; 30:1070-1071.

8. Poppenga, R.H. Herbal medicine: potential for intoxication and interactions with conventional drugs. Clin. Tech. Small. Anim. Pract. 2002; 17: 6-18.

9. Yaffe, S.J. and Aranda, J.V. Pharmacology in Paediatrics. In PL Munson, R.A Mueller, G.R Breese, Principles of Pharmacology: Basic Concepts and Clinical Applications, Chapman and Hall, New York. 1995; 1689-1709.

10. Tindimwebwa, G. and Dambisya, Y.M. A retrospective study of herbal intoxication in children at UGH. Abstracts of the 4th Eastern Cape Health Research Conference. Umtata. 2002; 25.

11. Dambisya, Y.M. and Tindimwebwa, G. The use of traditional remedies in children in communities around Umtata. Abstracts of the 4th Eastern Cape Health Research Conference. Umtata 2002;24.

12. Nazareth, I. and Mfenyana, K. Medical education in the community - the UNITRA experience. Med. Educ. 1999; 33:722-724.

13. Steenkamp, V. Toxicology of traditional remedies. Science in Africa (on line) February 2002. http://www.scienceafrica.co.zal 2002/february/toxic.htm.

14 Nyanzema, N.Z. Poisoning due to traditional remedies. Cent. Afr. J. Med. 1984; 30:80-83. 